\title{
Reconstruction of optical pathlength distributions from images obtained by a wide-field differential interference contrast microscope
}

\author{
E. B. VAN MUNSTER, * L. J. VAN VLIET $\dagger \&$ J. A. ATEN* \\ *Department of Radiotherapy, Academic Medical Center, University of Amsterdam, PO Box \\ 22700, 1100 DE Amsterdam, The Netherlands \\ $\dagger$ Pattern Recognition Group, Faculty of Applied Physics, Delft University of Technology, \\ Lorentzweg 1, 2628 CJ Delft, The Netherlands
}

Key words. DIC, Nomarski, interference, microscopy, CCD, image processing, analysis, reconstruction, optical pathlength, phase, transparent, living.

\begin{abstract}
Summary
An image processing algorithm is presented to reconstruct optical pathlength distributions from images of nonabsorbing weak phase objects, obtained by a differential interference contrast (DIC) microscope, equipped with a chargecoupled device camera. The method is demonstrated on DIC images of transparent latex spheres and unstained bovine spermatozoa. The images were obtained with a wide-field DIC microscope, using monochromatic light. After image acquisition, the measured intensities were converted to pathlength differences. Filtering in the Fourier domain was applied to correct for the typical shadow-cast effect of DIC images. The filter was constructed using the lateral shift introduced in the microscope, and parameters describing the spectral distribution of the signal-to-noise ratio. By varying these parameters and looking at the resulting images, an appropriate setting for the filter parameters was found. In the reconstructed image each grey value represents the optical pathlength at that particular location, enabling quantitative analysis of object parameters using standard image processing techniques. The advantage of using interferometric techniques is that measurements can be done on transparent objects, without staining, enabling observations on living cells. Quantitative use of images obtained by a wide-field DIC microscope becomes possible with this technique, using relatively simple means.
\end{abstract}

\section{Introduction}

Interference- and phase-contrast microscopy are imaging techniques invented more than 60 years ago to visualize

Correspondence to: Erik B. van Munster. Tel: +31 20 5664812; fax: +31 20 5664440; e-mail: e.b.vanmunster@amc.uva.nl

(c) 1997 The Royal Microscopical Society unstained transparent objects (Sirks, 1892; Zernike, 1935). The availability of lasers, scanning tables, charge-coupled device (CCD) cameras and computers in the last decades has permitted the development of various quantitative measuring systems for interferometric parameters (Brakenhoff, 1979; Svensson, 1957; te Meerman et al., 1986; Laffon et al., 1996). However, the use of interferometry remained limited to qualitative and morphological applications, with some exceptions (Barer et al., 1953; Davies et al., 1953; Grampp et al., 1960; James \& Dessens, 1962; Carlson \& Gledhill, 1966; Pluta, 1967; Sumner \& Robinson, 1976; Laffon et al., 1996). Somehow the various quantitative techniques never found widespread use in biology. This is partly due to the fact that in these systems, dedicated interferometric measuring set-ups were used, often involving scanning lasers, piezo-driven mirrors or rotating gratings, making these systems expensive and difficult to operate. Also, the focus of attention for quantitative applications has shifted towards fluorescence microscopy. In certain situations, however, there is still a need for quantitative measurements on cells, for instance analysing living cells, without the need to incorporate stains.

A set-up whereby a wide-field microscope is used, in combination with a CCD camera, has many advantages, apart from the fact that interference- and phase-contrast microscopes are already available in many laboratories. Once the images are recorded, interferometric parameters can automatically be determined by image processing. It is more efficient in this way to analyse large numbers of cells than with a nonimage-based interferometric measuring system. Much of the image processing already developed for quantitative fluorescence and absorption microscopy can easily be adapted. Before recordings of microscopical images can be used for quantitative applications, the intensities 
measured by the CCD camera need to be converted back to optical pathlengths.

In phase-contrast, the relation between optical pathlength and intensity in the formed image is very complicated and spatial frequency dependent (the halo effect) (Wolter, 1950; Bennet et al., 1951), thereby making phasecontrast images unfit for quantitative analysis.

This relation is simpler in interference-contrast microscopy, but here also some sort of artefact is introduced in the image (James, 1976). This is observed as a 'ghost image' either shifted laterally with respect to the original or superimposed as an out-of-focus blur. In differential interference contrast (DIC), the lateral shift is so small that the typical 'shadow-cast' appearance is formed. We will show that if the direction and the distance of the lateral shift are known, DIC images of weak phase objects, i.e. transparent objects that induce a phase shift smaller than $\pi / 2$, can be corrected for this effect. In this article we present and demonstrate a method to determine an optical pathlength distribution over an image, using a standard DIC microscope, a CCD camera and image processing software.

\section{Principles of differential interference contrast}

The phenomenon on which all interference microscopy is based is the interference of light waves. If two coherent light beams of the same wavelength and intensity are

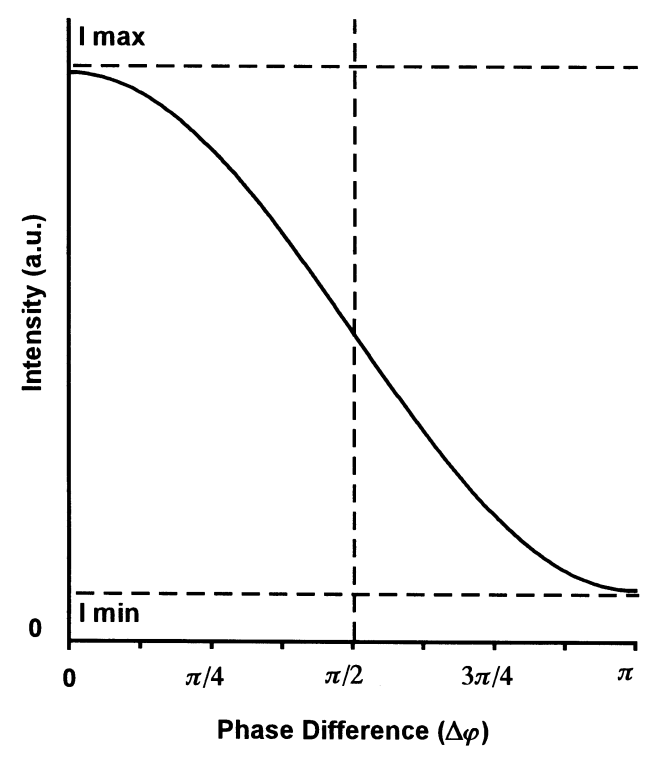

Fig. 1. The intensity of light after interference between two monochromatic coherent light waves. Maximum intensity is reached when the two waves are in phase $(\Delta \varphi=0)$, and minimum intensity if the waves are $\pi$ out of phase. If an additional phase shift (bias) of $\pi / 2$ is given, a small change in $\Delta \varphi$ will cause the largest variation in intensity, making maximum use of the dynamic range of the system. superimposed, the intensity of the resultant light is dependent on the phase difference $(\Delta \varphi)$ between the two beams. If the waves are in phase $(\Delta \varphi=0)$ constructive interference causes the amplitude to double; if the waves are out of phase $(\Delta \varphi=\pi)$ destructive interference occurs, and the resultant intensity is 0 . This is shown in Fig. 1 .

Differential interference contrast, or Nomarski, microscopy, was developed in the 1950s (Nomarski \& Weill, 1955). Here, the interference is achieved by splitting the incoming light into two components, shifted laterally over a very small distance (in the order of the wavelength of the light used), by a double refracting prism in the condenser. After passing through the object plane and the objective, the two components are recombined so that interference, as described above, takes place (Padawer, 1968; Allen et al., 1969; Cogswell \& Sheppard, 1992). An additional phase shift (bias) can be given to one of the beams, through a rotating filter in the condenser. Usually a bias of $\pi / 2$ is given, so that maximum use is being made of the dynamic range. A positive difference between the two beams will result in a lower intensity, and a negative difference will give a higher intensity (see Fig. 1).

If we look at a DIC image of a small object we can see how the lateral shift introduced by the microscope affects the image. Figure 2 is a CCD recording of a transparent latex sphere (Ø $460 \mathrm{~nm}$ ) observed through a DIC microscope

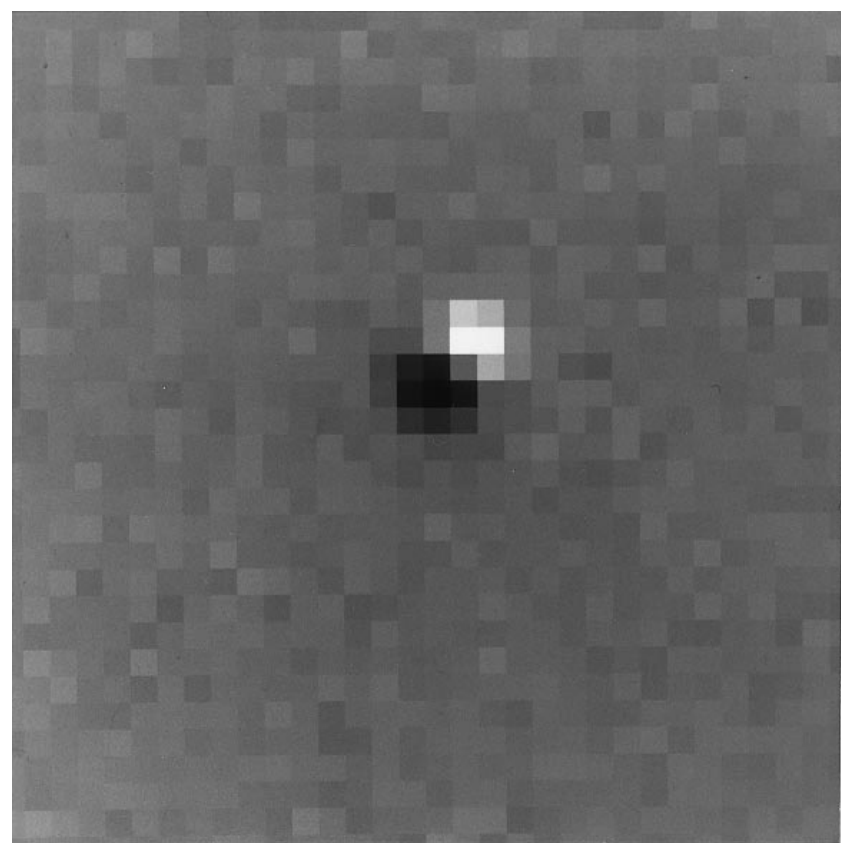

Fig. 2. An enlargement of a DIC recording of transparent latex spheres (Ø $460 \mathrm{~nm})$, in monochromatic light $(550 \mathrm{~nm})$, with bias set at $\pi / 2$. The lateral shift introduced by the microscope can be determined by measuring the distance between the centre of the bright spot and the centre of the dark spot. 


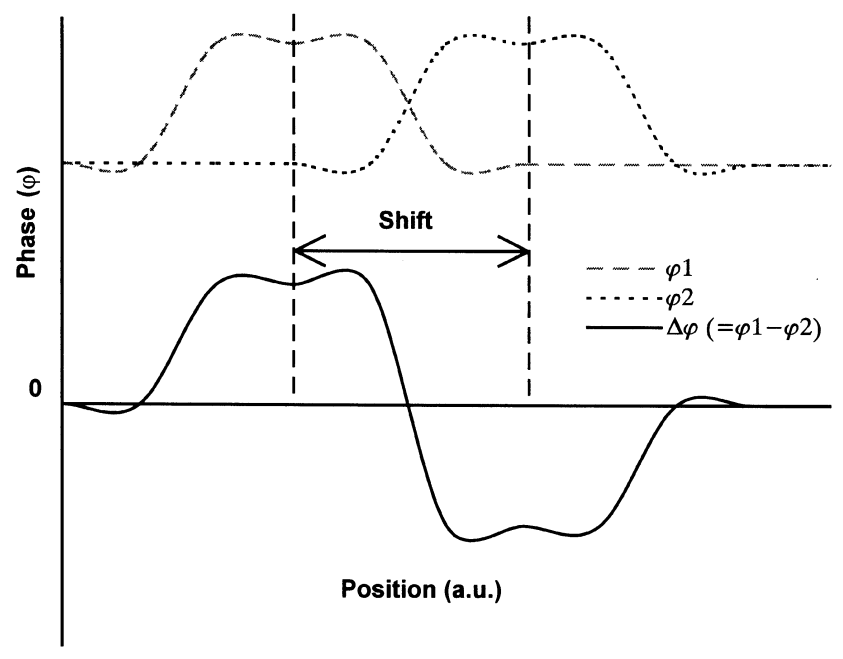

Fig. 3. A schematic view of image formation in a DIC microscope. $\varphi 1$ and $\varphi 2$ represent the two wavefronts resulting from one object, shifted with respect to each other as a result of the lateral shift introduced in the microscope. The difference between the two waves is converted to an intensity profile. If a bias of $\pi / 2$ is given, a positive phase difference will result in a lower intensity, and a negative phase difference in a higher intensity (see Fig. 1).

with bias set to $\pi / 2$. The 'double' image is formed as a result of the lateral shift. This is shown schematically in Fig. 3. Owing to the lateral shift there are two wavefronts formed. The intensity resulting from interference is dependent on the phase difference between these two wavefronts. If we subtract the two phase profiles to obtain this difference, a positive and a negative peak can be seen. Using a bias of $\pi /$ 2 , this results in a lower and a higher intensity for the two peaks, similar to that observed in Fig. 2.

The typical 'shadow-cast' effect observed when using a DIC microscope is also due to this lateral shift. The largest phase difference between the two beams is formed when one of the beams falls just within, and the other beam just outside, the object. This happens at the edges of objects, perpendicular to the shift. Opposite sides of the object will give rise to opposite phase differences, resulting in a bright edge on one side and a dark edge on the other side, giving the impression that objects are observed in relief.

\section{Materials and methods}

\section{The reconstruction algorithm}

A first step in reconstructing DIC images is the conversion of the measured intensities to phase differences. The intensity resulting from interference between two superimposed coherent monochromatic light waves can be described as

$$
I=\frac{1}{2} I_{\max } \cdot(1+\cos \Delta \varphi)
$$

with $I$ the measured intensity, $I_{\max }$ the maximum intensity and $\Delta \varphi$ the phase difference between the two waves (Born \& Wolf, 1986).

If we measure this intensity in an interference microscope, the maximum intensity is not constant over the image, because of uneven illumination. Furthermore, we do not see complete destructive interference when the waves are $\pi$ out of phase; always some residual light is measured. We found a better description of the intensity to be

$$
\begin{aligned}
I(x, y)= & I_{\min }(x, y)+\left[I_{\max }(x, y)\right. \\
& \left.-I_{\min }(x, y)\right] \cdot \frac{1}{2} \cdot[1+\cos (\Delta \varphi(x, y)+\delta)],
\end{aligned}
$$

with $I(x, y)$ the intensity measured at location $(x, y), I_{\min }(x$, $y)$ the residual intensity at destructive interference, $I_{\max }(x$, $y$ ) the intensity measured at constructive interference and $\delta$ the additional phase shift, or bias, given to one of the components.

When we look at Fig. 1 we can see that if we want to convert intensities back to phase differences, the best accuracy can be achieved in the steepest, most linear part of the curve. A bias of $\pi / 2$ will cause the phase difference to be centred around this point. If $-\pi / 2<\Delta \varphi<\pi / 2$, using $\delta=\pi / 2, \Delta \varphi$ can be calculated using Eq. (2):

$$
\Delta \varphi(x, y)=\arccos \left[2 \cdot \frac{I(x, y)-I_{\min }(x, y)}{I_{\max }(x, y)-I_{\min }(x, y)}-1\right]-\frac{\pi}{2} .
$$

It is important to notice that $\Delta \varphi(x, y)$ does not represent the phase at location $(x, y)$, but the difference between the phase at location $(x-\Delta x / 2, y-\Delta y / 2)$ and the phase at location $(x+\Delta x / 2, y+\Delta y / 2)$, where $(\Delta x, \Delta y)$ is the lateral shift introduced by the microscope. The second step in the reconstruction is to convert this 'differential' image $\Delta \varphi(x$, $y)$, to an image $\varphi(x, y)$, which does represent the phase at location $(x, y)$ :

$$
\Delta \varphi(x, y)=\varphi\left(x-\frac{1}{2} \Delta x, y-\frac{1}{2} \Delta y\right)-\varphi\left(x+\frac{1}{2} \Delta x, y+\frac{1}{2} \Delta y\right) .
$$

This can also be expressed in terms of a convolution:

$$
\Delta \varphi(x, y)=g(x, y) \otimes \varphi(x, y)
$$

with

$$
g(x, y)=\delta\left(x-\frac{1}{2} \Delta x, y-\frac{1}{2} \Delta y\right)-\delta\left(x+\frac{1}{2} \Delta x, y+\frac{1}{2} \Delta y\right) .
$$

From Eq. (5) we can see that calculating $\varphi(x, y)$ from $\Delta \varphi(x$, $y$ ) is equivalent to deconvolving $\Delta \varphi(x, y)$ using $g(x, y)$.

Our approach to perform this deconvolution is based on the property that convolution in the spatial domain corresponds to multiplication in the frequency domain:

$$
\Delta \Phi(u, v)=G(u, v) \cdot \Phi(u, v),
$$

where $\Delta \Phi(u, v), G(u, v)$ and $\Phi(u, r)$ are the two-dimensional Fourier transformations of $\Delta \varphi(x, y), g(x, y)$, and $\varphi(x, y)$, respectively, and $(u, v)$ the spatial frequencies $[-1 / 2 . .1 / 2]$. 


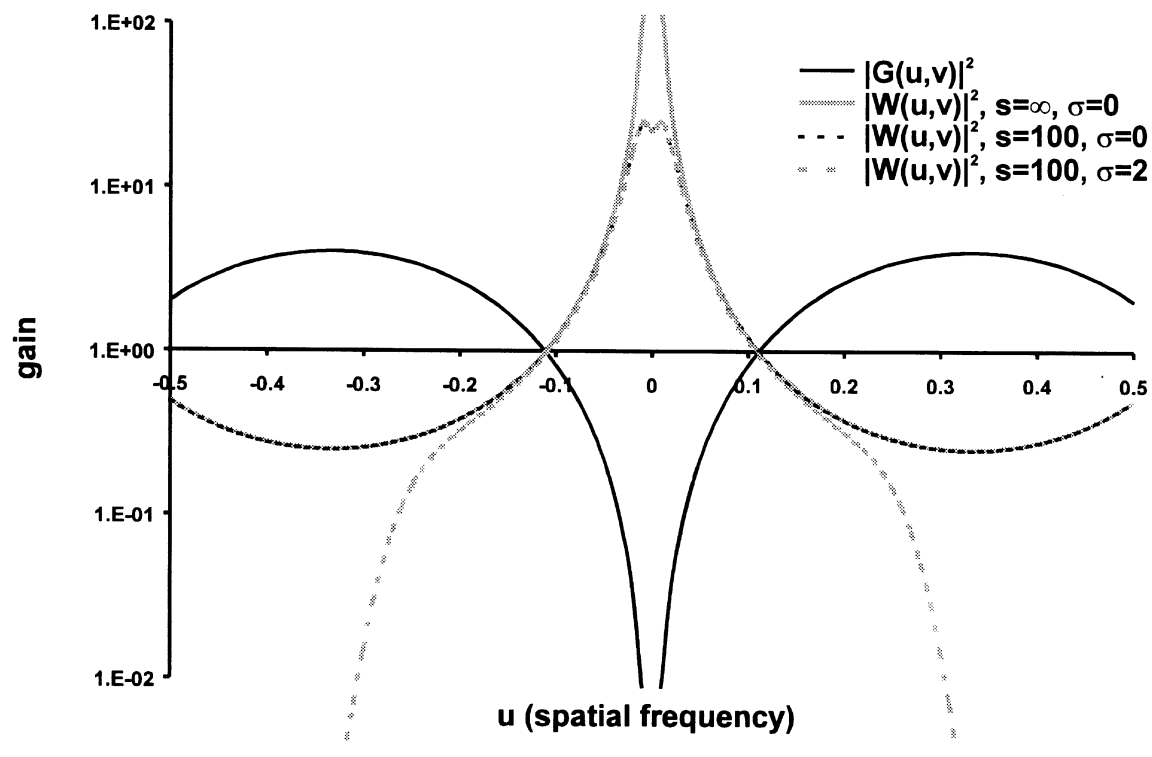

Fig. 4. The effect of a lateral shift $(\Delta x=1 \cdot 5$, $\Delta y=0)$ on the spectral contents of an image $(G(u, v))$, and the Wiener filter $(W(u, v))$, to correct for these effects. A lower value of $s$ will make the correction less strict, but is less sensitive to noise. $\sigma$ controls the inclusion of higher frequencies in the reconstructed image.
From Eq. (6) we calculate

$$
G(u, v)=2 j \sin (\pi \cdot(u \Delta x+v \Delta y)) .
$$

$|G(u, v)|^{2}$ as a function of $u$ is plotted in Fig. 4 for $\Delta x=1.5$, $\Delta y=0$.

The obvious solution to obtain $\Phi(u, v)$ would be to divide $\Delta \Phi(u, v)$ by $G(u, v)$. However, there will always be a noise component present in $\Delta \Phi(u, v)$, independent of $g(x, y)$, which has to be taken into account. Dividing by $G(u, v)$ also amplifies regions in the spectrum where noise is predominant over the signal, causing an amplification of noise. Furthermore, since we use an analytical model for $G(u, v)$, we run into trouble when $G(u, v)=0$. The optimal estimation for $\Phi(u, v)$, i.e. the solution that minimizes the noise for a given spectral signal-to-noise ratio distribution, can be found using Wiener filtering (Jansson, 1997):

$$
\Phi(u, v)=W(u, v) \cdot \Delta \Phi(u, v),
$$

with

$$
W(u, v)=\frac{G^{*}(u, v)}{|G(u, v)|^{2}+[1 / S N(u, v)]},
$$

called the Wiener filter, where $S N(u, v)$ is the spectral distribution of the signal-to-noise ratio.

We approximated $S N(u, v)$ by a two-dimensional circular Gauss curve centred at the middle $(u=0, v=0)$ of the spectrum:

$$
S N(u, v)=s \cdot \exp \left[-2 \pi^{2} \sigma^{2}\left(u^{2}+v^{2}\right)\right] .
$$

The Wiener filter, $W(u, v)$, is now described by four parameters: $\Delta x, \Delta y, s$ and $\sigma . \Delta x$ and $\Delta y$ are determined by the lateral shift introduced in the microscope, $s$ corresponds to the maximum signal-to-noise ratio and $\sigma$ describes the decrease towards higher spatial frequencies.
The effect of applying the Wiener filter in the Fourier domain becomes clear if we look at Fig. 4. Multiplying by $W(u, v)$ corrects for the effects introduced by the lateral shift $(G(u, v)$ in Fig. 4). The correct values for $s$ and $\sigma$ can be found by trying the filter on real images.

After multiplying $\Delta \Phi(u, v)$ with the Wiener filter, according to Eq. (9), $\varphi(x, y)$ can be calculated by inverse two dimensional Fourier transformation.

\section{Sample preparation}

Latex spheres (Ø $460 \mathrm{~nm}$, DOWlatex 41984, Serva, Germany), transparent at $550 \mathrm{~nm}$, were suspended in $100 \%$ ethanol, to a concentration of $1000 \mathrm{~mL}^{-1}$. A small drop of the mixture was put on a normal object slide and air dried. The spheres were immersed in phosphate-buffered saline (PBS) and were covered with a normal coverslip. Recordings were made immediately after preparation.

Bovine spermatozoa were used to test the reconstruction algorithm. They are transparent in visible light, and their maximal optical pathlength (when immersed in PBS and observed at $550 \mathrm{~nm}$ ) falls well within the $[-\pi / 2 . . \pi / 2]$ range because of their flatness $( \pm 0 \cdot 7 \mu \mathrm{m})$.

A straw $\left( \pm 10 \times 10^{6}\right.$ cells $)$ of cryopreserved bovine spermatozoa (Holland Genetics, Arnhem, The Netherlands) was thawed by immersing the straw in a $35^{\circ} \mathrm{C}$ water bath for $1 \mathrm{~min}$. The cells were suspended in $5 \mathrm{~mL}$ PBS and centrifuged at $400 \mathrm{~g}$ for $5 \mathrm{~min}$. The supernatant was removed, and the pellet resuspended in $10 \mathrm{ml}$ PBS. $0 \cdot 2 \mathrm{~mL}$ of the suspension was applied to an object slide by centrifuging (Cytospin II, Shandon, U.K.) at $90 \boldsymbol{g}$ for 10 min. After centrifuging, the cells were covered with a coverslip using PBS as mounting medium. Recordings were made immediately after preparation. 


\section{Recordings}

The slides were observed through a normal DIC microscope (Ortholux II, Leitz, Germany). A Leitz 25× DIC, (NA 0.5) objective was used for all observations. The bias was adjustable via a rotating polarizer in the condenser and was set at a phase shift of $\pi / 2$, except for the $I_{\max }$ and $I_{\min }$ images. A band-pass filter (MAD8-1, peak $550 \mathrm{~nm}$, width $8 \mathrm{~nm}$, Schott Glaswerke, Germany) was put in the light path of the microscope, between the lamp and the condenser. Recordings were made using a cooled CCD camera (HiSIS24, Lambert Instruments, The Netherlands), producing $512 \times 512$ 14-bit pixel images. $I_{\max }$ and $I_{\min }$ images needed for the conversion to phase differences were recorded prior to the recording of each image in the following way. The slide was scanned by eye for an area without cells (usually towards the edges of the coverslip), and recordings were made with bias set at 0 ( $I_{\max }$ image) and $\pi$ ( $\left(I_{\text {image }}\right)$, using the same exposure time as for the recording itself.

\section{Image processing}

The reconstruction algorithm, as described above, was written in $\mathrm{C}$ and implemented in SCIL-image (TNO/TPD, Delft, The Netherlands) running on a UNIX workstation (Silicon Graphics Indy, SGI, U.S.A.). After image acquisition, intensities measured with the CCD camera were converted to phase differences, pixel by pixel, according to Eq. (3), using the $I_{\max }$ and $I_{\min }$ images recorded with each image. During the conversion, checks were made to see if $\Delta \varphi$ fell within the $-\pi / 2 . . \pi / 2$ range, as required by Eq. (3). None of the pixels fell outside this range.

A recording of latex spheres was converted to phase differences in this way, and used to determine the lateral shift $(\Delta x, \Delta y)$ in the following way. The centre of the positive values in the $\Delta \varphi$ image (the bright spot in Fig. 2), and of the negative values (the dark spot in Fig. 2) was determined. This was done by calculating the average $x$ coordinate and the average $y$ coordinate. in a square $32 \times 32$-pixel window around the sphere image, weighted with $|\Delta \varphi(x, y)|$, using only the positive values, or only the negative values, respectively. The difference between these two locations was taken as $(\Delta x, \Delta y)$.

A $512 \times 512$-pixel image with complex pixel values was constructed using $\Delta x, \Delta y$, according to Eq. (8). $\Delta S N(u, v)$ was constructed according to Eq. (11). Several values for $s$ and $\sigma$ were tested. The imaginary part of $S N(u, v)$ was set to $0 . W(u, v)$ was constructed according to Eq. (10). A two-dimensional discrete Fourier transform was performed on $\Delta \varphi(x, y)$ to obtain $\Delta \Phi(u, v)$ and $\Delta \Phi(u, v)$ was multiplied with $W(u, v)$, according to Eq. (9). Finally, $\varphi(x, y)$ was obtained by an inverse two-dimensional discrete Fourier transform.

\section{Results}

A CCD recording of latex spheres was recorded and converted to phase differences. Five spheres were visible in the image. A $32 \times 32$-pixel enlargement of one of the spheres is shown in Fig. 2. This sphere was used to determine the lateral shift. After analysis we found $\Delta x=1 \cdot 751$ and $\Delta y=2 \cdot 381$. Wiener filtering, using $\Delta x=1 \cdot 751, \Delta y=2 \cdot 381, s=1 \times 10^{11}, \sigma=3 \cdot 84$, was performed on the same image. The result is shown in Fig. 5. As can be seen, the 'double' image due to the lateral shift disappears.

A recording of bovine spermatozoa is shown in Fig. 6 . After conversion to phase differences using the $I_{\min }$ and $I_{\max }$ images recorded with the image, a Wiener filter was applied with the same parameter used in Fig. 5. The result is shown in Fig. 7. The 'shadow-cast' effect has disappeared and image interpretation is much easier; the grey values in the image are a direct measure of the optical pathlength. The brighter a pixel in the reconstructed image, the higher the optical pathlength at that particular location.

To investigate the role of $s$ and $\sigma$, a section of Fig. 6 was reconstructed using different settings for these parameters. A small section of the reconstructions is shown in Fig. 8. As can be seen, the quality of the reconstruction depends very much on the choice of $s$ and $\sigma$. A lower $\sigma$ enhances details in the image, but below a certain value $( \pm \sigma=1.92)$ mostly noise is added (Fig. 8A-D). Too low a value for $s$ will cause darkening in the centre of larger objects, corresponding to a

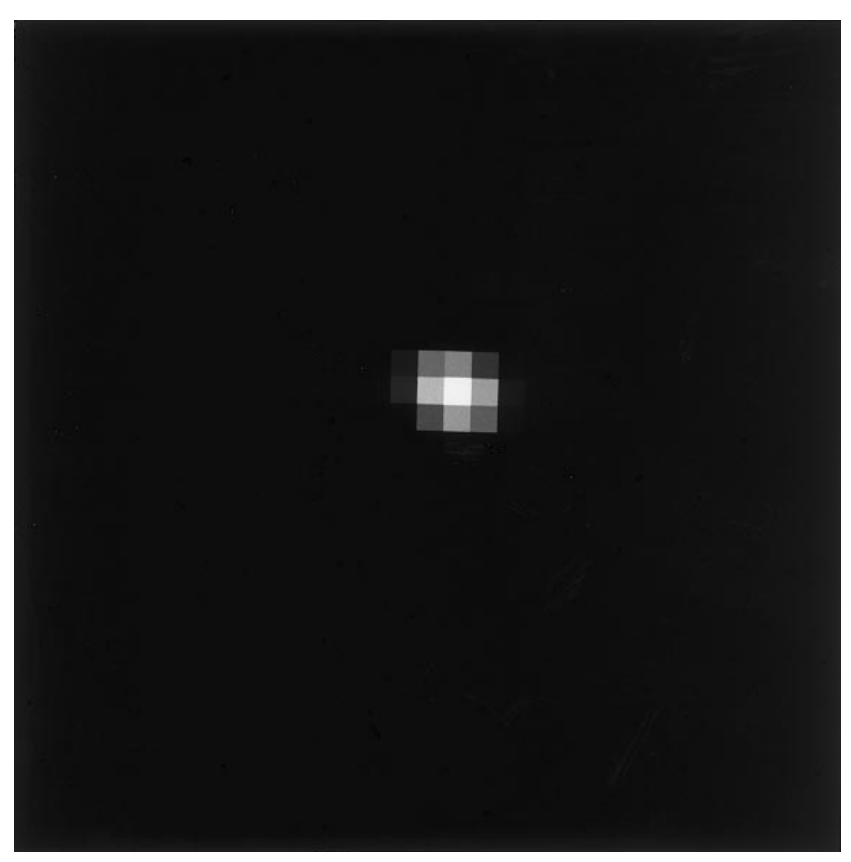

Fig. 5. A reconstruction of the DIC recording in Fig. 2. The image was reconstructed using $\Delta x=1 \cdot 752, \Delta y=-2 \cdot 381, s=1 \times 10^{11}$, $\sigma=3 \cdot 84$. 


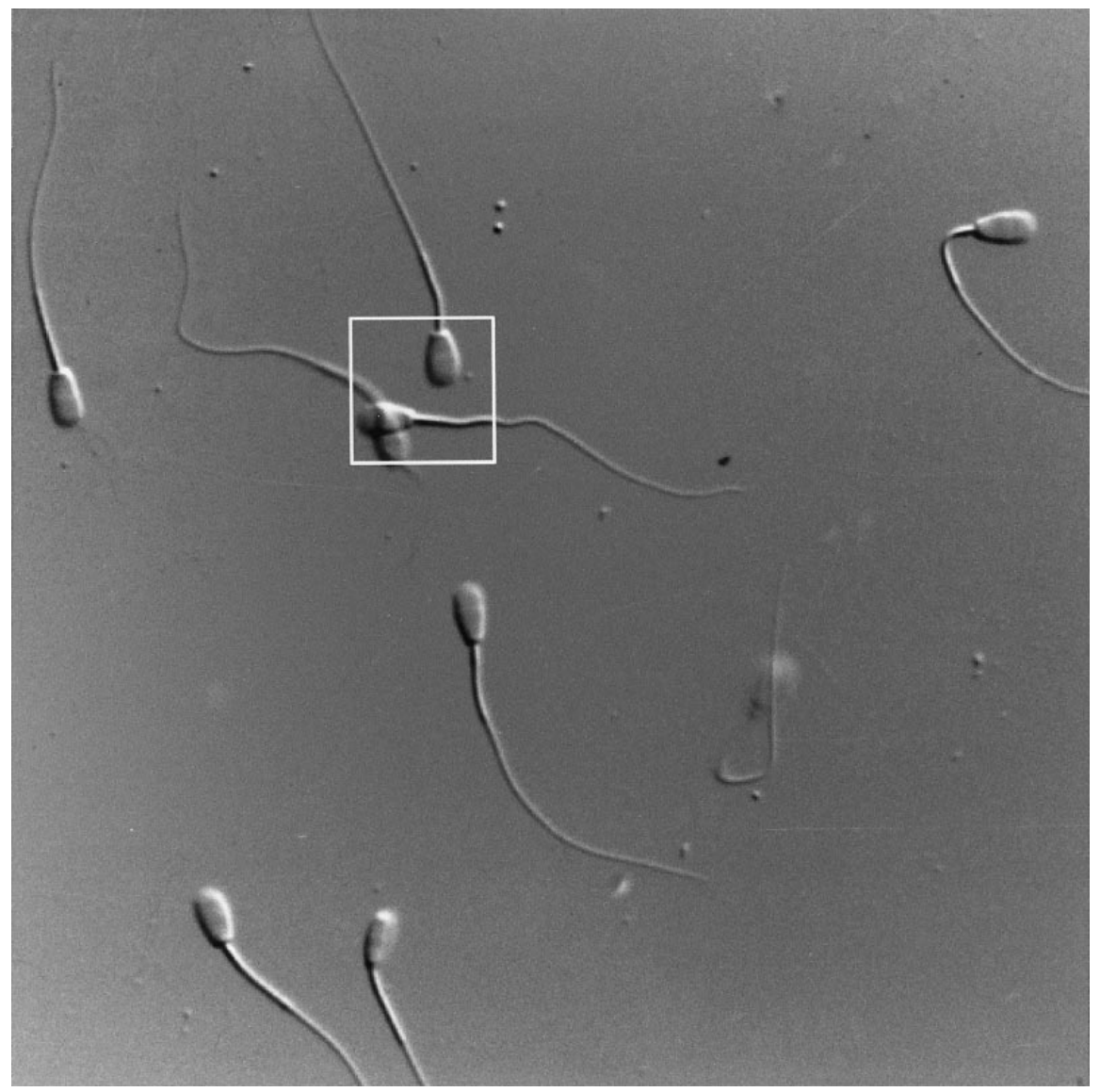

Fig. 6. A DIC recording of unstained bovine spermatozoa mounted in PBS, in monochromatic light $(550 \mathrm{~nm})$, with bias set at $\pi / 2$.

reduction of the low-frequency content of the image. This is clearly visible in Fig. 8(E). Alongside the overlapping of the two cells a dark edge is seen. When increasing $s$, this darkening disappears (Fig. 8E-H). A value too high for $s$, however, will cause stripes in the image, parallel to the direction of the lateral shift (Fig. 8H).

\section{Discussion and conclusion}

Looking at the reconstructed images it can be seen that the artefacts caused by the lateral shift, i.e. the shadow-cast effect, have disappeared. The quality of the image is very much dependent on the choice of $s$ and $\sigma$. The values $s=1 \times 10^{11}$ and $\sigma=3 \cdot 84$, as used in Figs. 5 and 7 , seemed to produce the best results, although this choice is purely subjective and based only on judgement by eye. The sensitivity of the reconstruction algorithm to the choice of parameters is important to bear in mind when considering quantitative application of the technique, when analysing interferometric data obtained from different reconstructed images, the parameters used in the reconstruction should be the same. Care should be taken that the camera position with respect to the microscope is not altered between recordings. A slight rotation of the camera will cause a change in the direction of the lateral shift in the recorded image.

The method used to reconstruct the DIC images, i.e. deconvolution, closely resembles other techniques for reconstruction of (deteriorated) microscopy images. Usually a recording of a small (fluorescent or absorbent) bead is used as a point spread function (PSF). The reconstruction is based on the assumption that the original was convolved with this PSF to produce the observed image. Deconvolution with the PSF is used to reconstruct the original. In fact this is similar to our approach, except that we use a model of the PSF (the convolution kernel $g(x, y)$ ), the parameters of which are based on a real recording. Instead of a model we could also use the recording of a sphere itself as the convolution kernel. In practice, however, the extra information included, the optical transfer function of the optical system, does not contribute to removing the artefacts introduced by the lateral shift. Furthermore, $S N(u, v)$, is based also on the effects of this optical transfer function. The approximation for $S N(u, v)$ used, the Gauss curve, is a crude one, based purely on judgement by eye of the resulting reconstructed images. Improvements will be made by trying to use a more realistic description of the shape of the optical transfer function of the microscope in the construction of 
Fig. 7. A reconstruction of Fig. 6 using $\Delta x=1 \cdot 752, \Delta y=-2 \cdot 381$, $s=1 \times 10^{11}, \sigma=3 \cdot 84$.

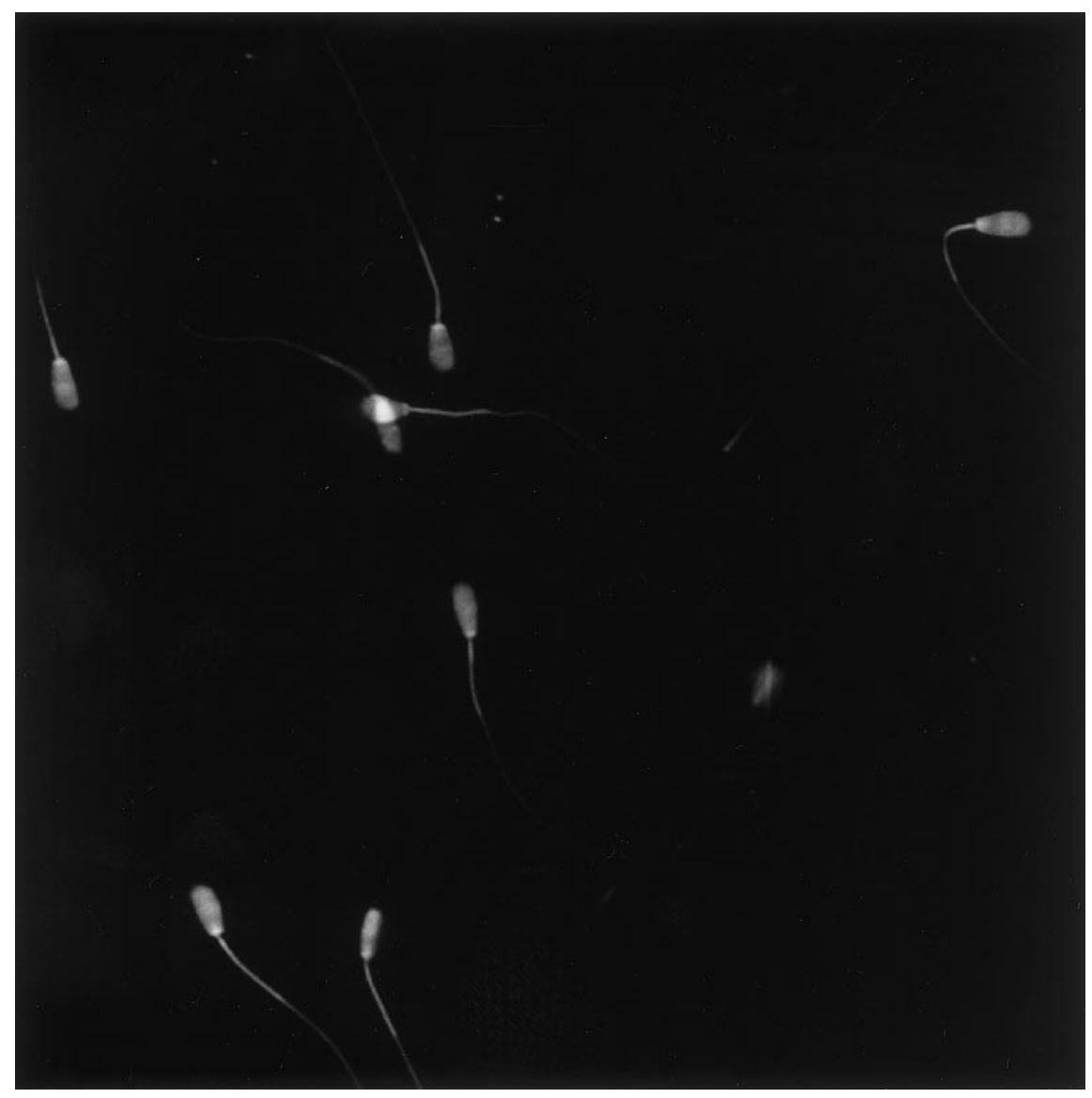

$S N(u, v)$ instead of the approximation used here. It should be pointed out that deconvolution by Wiener filtering assumes a linear response of the system. In interference microscopy, apart from the effects of the lateral shift, this is not the case. For this reason, nonlinear techniques will be more appropriate for further reconstruction (Preza et al., 1996, 1997).

The optical pathlength of an object, as seen in reconstructed images, is the product of the thickness and the difference in refractive index between the object and the surrounding medium. However, only the part of the object that lies within the depth of view of the objective lens contributes to the measured optical pathlength. When considering quantitative application one has to be sure that the complete object falls within this range. Future investigations will concentrate on the quantitative effects of out-of-focus contributions. With the system used here only objects thinner than $\pm 2 \mu \mathrm{m}$ can be quantified reliably.

An important application of the technique is the determination of DNA and protein content of cells. The integrated optical pathlength over a cell area is a measure of the solid content (DNA, protein) of that cell (Barer, 1952; Davies \& Wilkins, 1952). In our case, this is equivalent to calculating the sum of grey values of all the pixels of a cell in a reconstructed image. Solid contents of individual cells in a reconstructed image can thus be assessed, using relatively simple image processing. Large numbers of images can be recorded, reconstructed and analysed automatically in this way, using techniques similar to those already applied in quantitative absorption and fluorescence microscopy.

The reconstruction algorithm as presented and demonstrated in this paper is a good method to obtain the necessary images on which to perform further quantitative analysis, provided that the objects under investigation fall completely within the depth of view of the microscope used, and that their optical pathlength differences with the surrounding medium do not surpass $\pi / 2$. The fact that the method does not require any custom-built optics and used a wide-field existing DIC microscope in combination with a normal CCD camera makes the system easy to use and relatively cheap.

\section{Acknowledgments}

This project was financially supported by Holland Genetics, Arnhem, The Netherlands, project \#HGKW4. Thanks to G. J. te Meerman for his idea to use interferometry as quantitative measuring tool, and initiating this project. We would like to thank all the people at the Laboratory for 

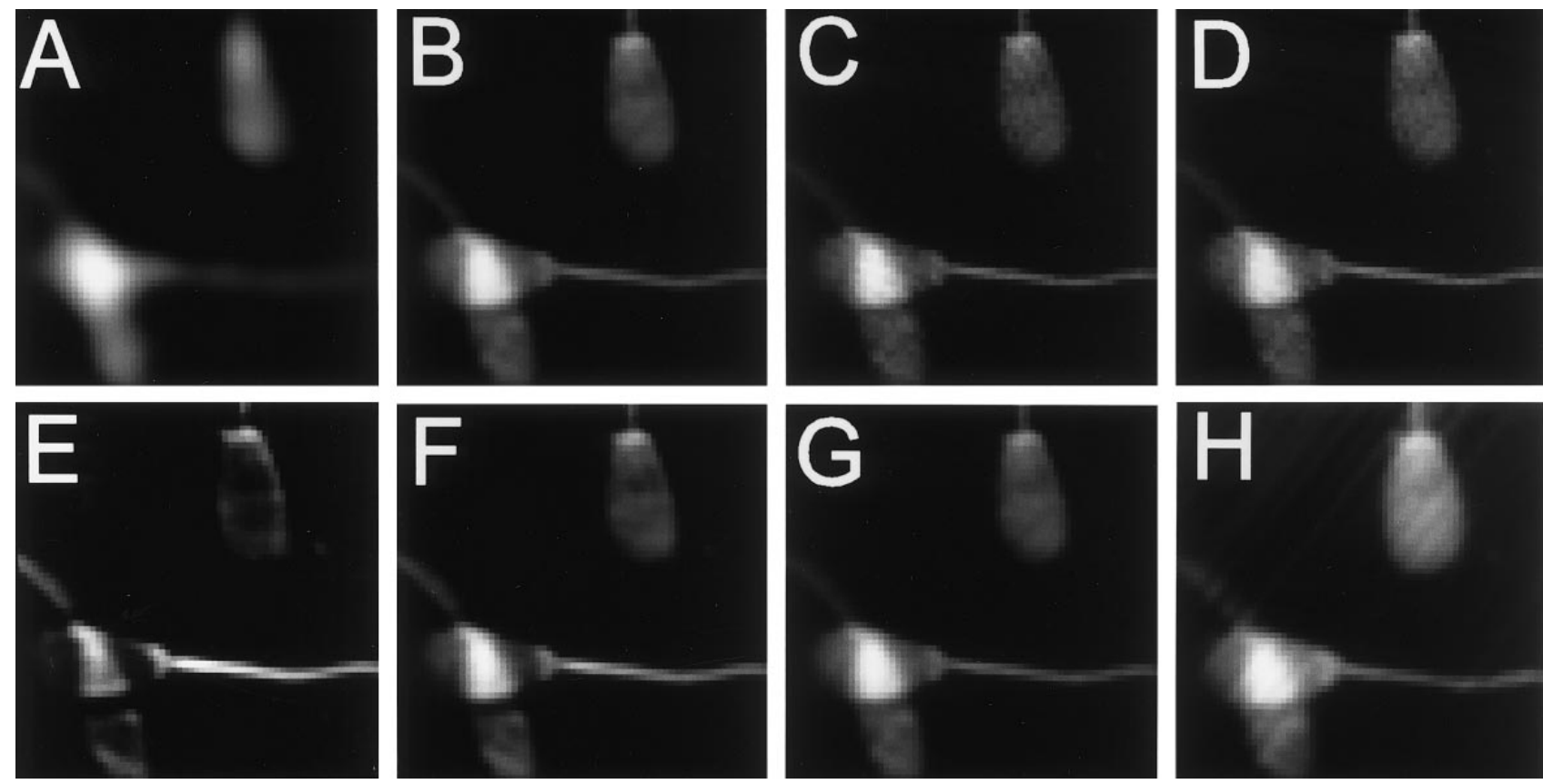

Fig. 8. A small section of reconstructions of Fig. 6 (the small square in Fig. 6) at various settings for $s$ and $\sigma(\Delta x=1 \cdot 752, \Delta y=-2 \cdot 381)$. A, $s=1 \times 10^{11}, \quad \sigma=11 \cdot 5 ; \quad \mathrm{B}, \quad s=1 \times 10^{11}, \quad \sigma=3 \cdot 84 ; \quad \mathrm{C}, \quad s=1 \times 10^{11}, \quad \sigma=1 \cdot 92 ; \mathrm{D}, \quad s=1 \times 10^{11}, \quad \sigma=0 \cdot 96 ; \mathrm{E}, \quad s=1 \times 10^{9}, \quad \sigma=3 \cdot 84$; F, $s=1 \times 10^{10}, \sigma=3 \cdot 84 ; \mathrm{G}, s=1 \times 10^{11}, \sigma=3 \cdot 84 ; \mathrm{H}, s=1 \times 10^{13}, \sigma=3 \cdot 84$.

Radiobiology who took the time to read and comment on the manuscript.

\section{References}

Allen, R.D., David, G.B. \& Nomarski, G. (1969) The ZeissNomarski differential interference equipment for transmitted light microscopy. Z. Wiss. Mikrosk. 69, 193-221.

Barer, R. (1952) Interference microscopy and mass determination. Nature, 169, 366-367.

Barer, R., Ross, K.F.A. \& Tkaczyk, S. (1953) Refractometry of living cells. Nature, 171, 720-724.

Bennet, A.H., Jupnik, H., Osterberg, H. \& Richards, O.W. (1951) Phase Microscopy, Principles and Applications. John Wiley, New York.

Born, M. \& Wolf, E. (1986) Principles of Optics, 6th edn. Pergamon Press, Oxford.

Brakenhoff, G.J. (1979) Imaging modes in confocal scanning light microscopy (CSLM). J. Microsc. 117, 233-242.

Carlson, L. \& Gledhill, B.L. (1966) Studies on the dry mass of bull spermatozoal heads. Exp. Cell Res. 41, 376-384.

Cogswell, C.J. \& Sheppard, C.J.R. (1992) Confocal differential interference contrast (DIC) microscopy: including a theoretical analysis of conventional and confocal DIC imaging. J. Microsc. $165,81-101$.

Davies, H.G., Engstrom, A. \& Lindstrom, B. (1953) A comparison between the X-ray absorption and optical interference methods for the mass determination of biological structures. Nature, 172, 1041.

Davies, H.G. \& Wilkins, M.H.F. (1952) Interference microscopy and mass determination. Nature, 169, 541.
Grampp, W., Hallen, O. \& Rosengren, B. (1960) Mass determination by interference- and x-ray microscopy. Exp. Cell Res. 19, 437-442.

James, J. (1976) Light Microscopic Techniques in Biology and Medicine, Martinus Nijhoff Medical Division, The Hague.

James, J. \& Dessens, H. (1962) Immersion-refractometric observations on the solid concentration of erythrocytes. J. Cell Comp. Phys. 60, 235-241.

Jansson, P.A. (ed.) (1997) Deconvolution of Images and Spectra, 3: Traditional Linear Deconvolution Methods. Academic Press, San Diego.

Laffon, E., Dilhaire, S., Leveque, J.L. \& Corcuff, P. (1996) An improved technique for optical interferometric imaging of isolated cells. Cytometry, 24, 93-96.

Meerman, G.J.te, Anders, G.J.P.A. \& Wiersma, J. (1986) Raster images and measurement with a Vickers M86 scanning microdensitometer and interferometer by computer control. J. Microsc, 141, 199-211.

Nomarski, G. \& Weill, A.R. (1955) Application à la métallographie des méthodes interférentielles à deux ondes polarisées. Rev. Metall. 2, 121-128.

Padawer, J. (1968) The Nomarski interference microscope; an experimental basis for image interpretation. J. R. Microsc. Soc. 88, 305-349.

Pluta, M. (1967) On the microinterferometric determination of the dry mass of non-plate cells. Folia Histochem. Cytochem. (Krakow), 5, 409-413.

Preza, C., Snyder, D. \& Conchello, J. (1996) Imaging models for three-dimensional transmitted-light DIC microscopy. SPIE Proc. 2655, 245-257.

Preza, C., Snyder, D.L. \& Conchello, J. (1997) Image reconstruction 
for three-dimensional transmitted-light DIC microscopy. SPIE Proc. 2984, 220-231.

Sirks, J.L. (1892) Een Interferentie Mikroskoop. Natuur en Geneeskundig. Congres 1892, pp. 92-98.

Sumner, A.T. \& Robinson, J.A. (1976) A difference in dry mass between the heads of X- and Y-bearing human spermatozoa. J. Reprod. Fertil. 48, 9-15.
Svensson, G. (1957) A scanning interference microphotometer. Exp. Cell Res. 12, 406-409.

Wolter H. (1950) Experimentelle und theoretische Untersuchungen zur Abbildung nichtabsorbierende Objekte. Ann. Phys. 6, 33-53.

Zernike, F. (1935) Das Phasenkontrastyerfahren bei der mikroskopischen Beobachtung. Z. Tech. Phys. 1, 454-457. 